Original article

\title{
Socioeconomically Disadvantaged Groups and Metabolic Syndrome in European Adolescents: The HELENA Study
}

Isabel Iguacel, Ph.D. ${ }^{\text {a, b,c, d, } 1, *}$, Claudia Börnhorst, Ph.D. ${ }^{e}$, Nathalie Michels, Ph.D. ${ }^{\text {f }}$,
Christina Breidenassel, Ph.D. ${ }^{\text {g,h }}$, Jean Dallongeville, Ph.D. ${ }^{\text {i }}$, Marcela González-Gross, Ph.D. ${ }^{\text {h,j }}$, Frédéric Gottrand, Ph.D. ${ }^{\text {, }}$, Anthony Kafatos, Ph.D. ${ }^{\text {, }}$ Eva Karaglani, Ph.D. ${ }^{\mathrm{m}}$, Mathilde Kersting, Ph.D. ${ }^{\mathrm{g}}$, Stefaan de Henauw, Ph.D. ${ }^{\text {f }}$, Christina-Paulina Lambrinou, Ph.D. ${ }^{1}$, Lorenza Mistura, Ph.D. ${ }^{\text {, }}$ Denes Molnár, Ph.D. ${ }^{\circ}$, Esther Nova, Ph.D. ${ }^{p}$, Marc J. Gunter, Ph.D. ${ }^{q}$, Alejandro de la O Puerta, Ph.D. ,

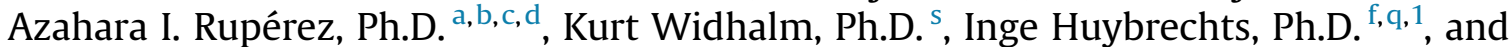
Luis A. Moreno, Ph.D. ${ }^{\text {a,b,c, d }}$

${ }^{a}$ GENUD (Growth, Exercise, NUtrition and Development) Research Group, Faculty of Health Sciences, Deparment of Physiatry and Nursing, University of Zaragoza, Zaragoza, Spain

${ }^{\mathrm{b}}$ Instituto Agroalimentario de Aragón (IA2), Zaragoza, Spain

${ }^{\mathrm{c}}$ Instituto de Investigación Sanitaria Aragón (IIS Aragón), Zaragoza, Spain

${ }^{\mathrm{d}}$ Centro de Investigación Biomédica en Red de Fisiopatología de la Obesidad y Nutrición (CIBERObn), Madrid, Spain

e Department of Biometry and Data Management, Leibniz Institute for Prevention Research and Epidemiology -BIPS, Bremen, Germany

${ }^{\mathrm{f}}$ Department of Public Health, Ghent University, Ghent, Belgium

${ }^{\mathrm{g}}$ Departement of Nutrition - Humannutrition, Rheinische Friedrich-Wilhelms-Universität Bonn, Bonn, Germany

${ }^{\mathrm{h}}$ ImFine Research Group, Department of Health and Human Performance, Facultad de Ciencias de la Actividad Fisica y del Deporte-INEF, Universidad Politecnica de Madrid, Madrid, Spain

${ }^{\mathrm{i}}$ Department of Epidemiology and Public Health, Institut Pasteur de Lille, Lille, France

${ }^{\mathrm{j}}$ Department of Health and Human Performance, Facultad de Ciencias de la Actividad Fsica y del Deporte-INEF, Universidad Politecnica de Madrid, Madrid, Spain

${ }^{\mathrm{k}}$ Inserm U995, IFR114, Faculty of Medicine, Universit de Lille2, Lille, France

${ }^{1}$ Preventive Medicine and Nutrition Clinic, University of Crete, Heraklion, Greece

${ }^{\mathrm{m}}$ Department of Nutrition and Dietetics Harokopio University of Athens, Athens, Greece

${ }^{\mathrm{n}}$ Council for Agricultural Research and Economics, Research Centre for Food and Nutrition, Rome, Italy

${ }^{\circ}$ Department of Paediatrics, University of Pecs, Pecs, Hungary

${ }^{\mathrm{p}}$ Immunonutrition Group (Metabolism and Nutrition Department) -Institute of Food Science, Technology and Nutrition, Spanish National Research Council (ICTAN-CSIC), Madrid, Spain

${ }^{\mathrm{q}}$ International Agency for Research on Cancer, World Health Organization, Lyon, France

${ }^{\mathrm{r}}$ Department of Physiology, Faculty of Medicine, University of Granada, Granada. Spain

${ }^{s}$ Department of pediatrics, University of Vienna, Vienna, Austria

Article history: Received October 28, 2019; Accepted May 8, 2020

Keywords: Metabolic syndrome; Socioeconomic disadvantages; Socioeconomic status; Obesity; adolescents; Modifiable lifestyle indicators

\section{A B S T R A C T}

Purpose: Psychosocial stressors derived from socioeconomic disadvantages in adolescents can result in higher risk of metabolic syndrome (MetS). We aimed to examine whether socioeconomic disadvantages were associated with MetS independent of lifestyle and whether there was a doseresponse relationship between the number of cumulated socioeconomic disadvantages and risk of MetS.

\section{IMPLICATIONS AND} CONTRIBUTION

Maternal education was the most important determinant of adolescent metabolic syndrome risk

\footnotetext{
Conflicts of interest: The authors have no conflicts of interest to disclose.

* Address correspondence to: I. Iguacel, Universidad de Zaragoza, Zaragoza, Aragon, Spain.
}

E-mail address: iguacel@unizar.es (I. Iguacel).

1 These authors contributed equally and share the last authorship. 
Methods: This study included 1,037 European adolescents (aged 12.5-17.5 years). Sociodemographic variables and lifestyle were assessed by self-reported questionnaires. Disadvantaged groups included adolescents with low-educated parents, low family affluence, migrant origin, unemployed parents, and nontraditional families. MetS risk score was calculated as the sum of sexand age-specific z-scores of waist circumference, blood pressure, lipids, and insulin resistance. Linear mixed-effects models adjusted for sex, age, pubertal status, and lifestyle were used to study the association between social disadvantages and MetS risk score.

Results: Adolescents with low-educated mothers showed a higher MetS score (.54 [.09-.98]; $\beta$ estimate and $99 \%$ confidence interval) compared to those with high-educated mothers. Adolescents who accumulated more than three disadvantages (.69 [.08-1.31]) or with missing information on disadvantages (.72 [.04-1.40]) had a higher MetS risk score compared to nonsocioeconomically disadvantaged groups. Stronger associations between socioeconomic disadvantages and MetS were found in male than in female adolescents.

Conclusions: Adolescents with low-educated mothers or with more than three socioeconomic disadvantages had a higher MetS risk, independent of lifestyle, potentially due to higher psychosocial stress exposure. Policy makers should focus on improving low-educated familiesa and more disadvantaged families' knowledge on nutrition and physical activity to help them cope better with stress.

(c) 2020 Society for Adolescent Health and Medicine. All rights reserved. and the association was independent of lifestyle. Other socioeconomic disadvantages seemed not to be associated with a higher risk, though a doseresponse relationship existed between the number of social disadvantages and metabolic syndrome risk. Loweducated families should be targeted to tackle health disparities.
The metabolic syndrome (MetS), defined as a cluster of metabolic abnormalities including abdominal obesity, hypertension, insulin resistance (IR), elevated triglyceride (TG), and reduced high-density lipoprotein cholesterol (HDL-C) circulating concentrations, is a major public health problem [1]. Having one of the components of MetS increases the risk of developing MetS in the future and can lead to a high lifetime burden of cardiovascular disease risk. Early detection of MetS components could enable targeted interventions to reduce the health and economic burden of cardiovascular diseases [2]. In 2018, the prevalence of MetS worldwide was estimated at 25\% [3] in adults and $10 \%$ in adolescents, with an increasing trend in the last decades [4]. This estimation differs widely by sex, age, race, ethnicity, region, and the definition of the MetS used [5].

Socioeconomic status (SES) is an important determinant of MetS. Previous studies on SES and MetS [6] have reported an inverse association, particularly in women [7]. Even during childhood, a low SES seems to predict a higher risk of MetS in adulthood independently of conventional childhood risk factors and the person's SES in adulthood [8]. In addition to classical SES indicators (including educational level, occupation, and income), there are other vulnerable groups (referred to hereafter as socioeconomically disadvantaged groups) that have not been extensively investigated but have been recently linked with higher MetS risk (or some of its components) independent of SES. These include adolescents whose parents are migrants, adolescents from nontraditional families, and adolescents with unemployed parents [9-11].

Socioeconomically disadvantaged groups often have adverse health behaviours that increase the risk of MetS such as the consumption of energy-dense foods, a highly sedentary lifestyle, lower physical activity (PA) levels, smoking, and heavy drinking [12]. These disadvantaged groups are more exposed to obesogenic environments [13] and they face more psychosocial stress derived from these social vulnerabilities. Psychologically stressful family environments and lack of access to social capital, including social support, might explain the association between childhood disadvantages and adult health [14]. In particular, social disadvantages can create a state of chronic stress that increases the activity of the hypothalamic-pituitary-adrenal axis leading to hypercortisolism and contributing to the development of several features of MetS [15].

We aimed to investigate the association between socioeconomic disadvantages and MetS risk score independent of lifestyle in a large cohort of European adolescents. Our hypothesis was that the effect of socioeconomic factors on MetS risk is independent of lifestyle, since there are other factors (e.g. stress derived from these socioeconomic disadvantages) that may explain this association. During adolescence, stress and many negative lifestyle behaviours intensify [16]. Moreover, we wanted to test which socioeconomic disadvantages (low-educated parents, low family affluence, migrant origin, unemployed parents, living in a nontraditional family) are the strongest MetS predictors.

\section{Methods}

\section{Population}

The current investigation used data from the Healthy Lifestyle in Europe by Nutrition in Adolescence Cross-Sectional Study (HELENA-CSS). The HELENA study is a cross-sectional multicenter study conducted between 2006 and 2007 in 10 European cities of more than 100,000 inhabitant each, located in separated geographical points in Europe (Athens and Heraklion, Dortmund, Ghent, Lille, Pecs, Rome, Stockholm, Vienna, and Zaragoza). The cities were selected by convenience based on the location of the partners in the HELENA study consortium. As a requirement, each city had a population with a high diversity in cultural background and socioeconomic situations; and the presence of an active research group assuring sufficient expertise and resources to successfully perform studies in adolescents [17]. The total sample included 3,528 adolescents, aged 12.5-17.5 years, from which one third were randomly included in the subsample that was selected for blood collection resulting in a total of 1,089 adolescents (Supplemental Table S1 compares the sample used 
in the study with the sample that had missing values for the MetS risk score). Adolescents were excluded from the database if they met one or more of the following exclusion criteria: age $<12.5$ or $\geq 17.5$ years; no measurement of weight and/or height; completion of $<75 \%$ of the tests; participation in another clinical trial; or contracting an acute infection during the week prior to the examination.

Adolescents and parents or legal guardians gave written informed consent for examinations and data collection for themselves and their children, respectively. Ethical approval was obtained from the research ethics authority of each participating center.

\section{Sociodemographic indicators and disadvantages scoring}

To define and capture socioeconomically disadvantaged groups $[18,19]$ within the sample, the following information was collected using a questionnaire completed by the adolescent participants.

Mother's and father's education was categorized as low education or medium/high education (higher secondary and higher education/university degree) [18,19].

Family affluence was based on the number of cars and computers owned by the household, the presence/absence of an Internet connection and whether adolescents had their own bedroom. A score of $0-3$ was coded as a low family affluence and 4-8 as a medium or high [19].

Family structure: adolescents from "traditional families" lived with both biological parents while those from nontraditional families were defined as those not living with their biological parents [18].

Origin of parents: a migrant background was assumed if one or both parents were born in a country different from the one where the study took place $[18,19]$.

Employment status: children with unemployed parents were those whose mother or father was unemployed, or those living on social assistance or welfare [18,19].

We calculated a total socioeconomic disadvantage score by summing the number of indicators of socioeconomic disadvantages a child was exposed to (low maternal education, low paternal education, low family affluence, nontraditional family, migrant, unemployed) [20,21]. This score ranged from 0 to six and was divided into four categories (3-6 disadvantages, 2, 1, and no disadvantages).

\section{Biochemical/clinical indicators and MetS scoring}

After biospecimen collection and examinations at the children's school, the following indicators were measured and assessed:

Waist circumference was used as an indicator of central adiposity, and it was measured in triplicate at the midpoint between the lowest rib and the iliac crest, using an anthropometric tape (SECA 200) [22].

Systolic blood pressure (SBP) and diastolic blood pressure (DBP) - were measured with an automatic oscillometric device (OMRON M6). Two measurements were taken with a 5-minute interval. The lowest value of the two recordings of systolic and diastolic measurement was selected.

Blood samples were taken by a nurse or medical doctor after 10 hours of fasting. For this study, glucose and HOMA-IR were selected as glucose indicators, whereas HDL and TG were taken as indicators of lipids. A more detailed description of the blood analysis has been previously reported elsewhere [23].

Pubertal stage was determined using Tanner's five-stage scale [24] during a clinical examination of each adolescent at their school. This information along with adolescent's age and sex were covariates in this study.

For each of the MetS risk components, sex- and age-specific z-scores were calculated. Following the definition of Ahrens et al. [25], a MetS score was calculated as the sum of sex- and age-specific z-scores of waist circumference, HOMA-IR index, mean of z-scores of SBP and DBP, and mean of z-score of HDL-C multiplied by -1 and $z$-score of TG. A higher score indicates poorer metabolic health.

\section{Lifestyle indicators}

Information on each adolescent's lifestyle factors was collected using self-report questionnaires.

PA was assessed using the question "Are you physically active for at least 1 hour each day?" Responses were categorized as: "Yes, for more than 6 months", "Yes, for 6 months or less," or "No".

Diet Quality Index for Adolescents

Dietary intake was assessed by two, nonconsecutive $24 \mathrm{~h}$ recalls. A Diet Quality Index for Adolescents (DQI-A) score was calculated by considering dietary quality, diversity, and equilibrium. Dietary quality expressed whether the adolescent made the optimal food quality choices within a food group. Dietary diversity described the degree of variation in the diet. Dietary equilibrium was calculated as the difference between an adequacy score (percentage of food groups with intake above the minimum recommended intake) and the excess component (percentage of food groups exceeding the upper level of the recommended intake).

DQI-A was calculated as the mean of dietary quality, diversity, and equilibrium and could range from $-33 \%$ to $100 \%$, with higher values reflecting a higher diet quality.

More detailed information about the DQI-A can be found elsewhere [26]. Using the median value of our sample, we categorized adolescents as showing a low DQI-A ( $\leq$ median) or high DQI-A (>median, with median $=54.66$ ).

Smoking status was assessed by: "Have you ever smoked tobacco?" and "if yes, how often do you smoke tobacco at present?". Those who reported "yes" were coded as "smokers". Participants that reported having smoked in the past but not currently were recoded as "ever smoker". Participants who never smoked tobacco were coded as "nonsmokers".

Alcohol intake: Adolescents reported consumption of alcoholic beverages in two $24 \mathrm{~h}$ recalls and were labelled as those who consumed alcohol in any of the two recalls and those who did not.

\section{Statistical analyses}

For descriptive analyses, chi-square tests were used to examine differences in the study population by adolescent's MetS risk (at risk vs. not at risk). Linear mixed-effects models were applied to study the associations between the six disadvantaged groups and MetS risk score including a random school and a random country effect to account for the clustered study design. Linear mixed-effects models were also used to assess the association between socioeconomic disadvantages and each of 
the MetS risk components, separately. Basic models were adjusted for age, sex, and Tanner stage. Fully adjusted models were additionally adjusted for lifestyle factors (PA, DQI, smoking, and alcohol intake) to assess the effect of social disadvantages not being mediated through lifestyle factors. Social vulnerabilities were also adjusted for parental education and family affluence to assess whether the effect was independent of classical SES indicators. For all our models, the nonvulnerable group was taken as a reference value.

As respondents with missing socioeconomic and lifestyle information may not be a random subset of the population-based survey, excluding these records from analyses may bias study results [27]. To avoid this issue, missing information/values in socioeconomic variables, vulnerability indicators, and lifestyle factors were coded as a separate category.

After excluding adolescents with missing values for MetS risk score and any of the components of MetS, this analysis included 1,037 adolescents (Supplemental Figure S1). The main characteristics of the subjects in both samples did not differ substantially based on comparison of the study sample with the sample that had missing values for the MetS risk score (Supplemental Table S1).

In order to test the robustness of our results several sensitivity analyses were conducted for the available sample size: (1) including a categorical outcome of MetS with different definitions of the MetS risk; (2) estimating all models stratified by sex; and (3) using family affluence as a continuous variable. Particularly for the first analysis, a dichotomous MetS variable was defined as having at least one of these conditions: abdominal obesity, hypertension, HOMA-IR, elevated TG, and reduced HDL$C$ (instead of three given the low number of adolescents diagnosed with MetS in our sample) according to the World Health Organization cutoffs [28]. Further analyses included the following definitions: AHA pediatric (American Heart Association), International Diabetes Federation, and NCEP-ATP (National Cholesterol Education Program Adult Treatment Panel III) (results not shown). For more information on these definitions, please refer to the study by Vanlancker et al.[28].

Statistical significance level was set at $p<.01$ to account at least partially for multiple testing. Analyses were performed using the Statistical Package for the Social Sciences (version 24.0; SPSS) [29].

\section{Compliance with ethical standards}

All procedures performed in studies involving human participants were in accordance with the ethical standards of the institutional and/or national research committee and with the 1964 Helsinki declaration and its later amendments or comparable ethical standards.

\section{Results}

\section{Description of the study population}

Table 1 shows the distribution of predictors and covariates stratified by adolescent's MetS risk. We observed differences in MetS risk by age groups, sex, country, alcohol intake, parent's education, and number of disadvantages (chi-square, $p<.05$ ). The percentage of adolescents at risk of MetS was higher for older adolescents, males, in Germany (while Sweden had the lowest risk), those who drank alcohol, those with a lower parental education, and with a higher accumulated number of disadvantages. Adolescents with a lower parental education and a nontraditional family structure had lower PA and DQI and a more frequent use of alcohol and smoking (Supplemental Table S1).

Associations between socioeconomic disadvantages and adolescent MetS risk scores or component risk scores

Table 2 presents $\beta$ estimates ( $\beta$ ) and 99\% confidence intervals (CI) for models assessing the associations between the six socioeconomically disadvantaged groups and adolescent's MetS risk score. In basic-adjusted models (not adjusted for lifestyle and therefore showing total effect of social vulnerabilities mediated through lifestyle), adolescents whose mother had a low education ( $\beta .54$ [99\% CI .09-.98]) had a significantly higher MetS score compared to the adolescents whose mothers had a medium or high education. Also, adolescents whose fathers did not report their education level had a higher MetS score ( $\beta$.74 [99\% CI .01-1.48]) compared to adolescents whose fathers reported to have a medium or high education. Effect estimates were attenuated when social vulnerabilities were additionally adjusted for maternal education and family affluence (results not shown).

Table 3 presents $\beta$ estimates and 99\% CI results for the associations between socioeconomic disadvantages and each component of the adolescent's MetS risk score (HOMA-IR, BMI, SBP, DBP, HDL, and TG). In basic-adjusted models, adolescents with low-educated mothers had a higher HOMA-IR ( $\beta .19$ [99\% CI $.01-.38])$, and TG ( $\beta .18$ [99\% CI .01-.36]) and lower HDL $(\beta-.17$ $[99 \% \mathrm{CI}-.37,-.00])$. Adolescents whose father did not report any information about their education had higher BMI ( $\beta$.34 [99\% CI $.01-.68])$.

Table 4 displays $\beta$ estimates and $99 \% \mathrm{CI}$ for the associations between the accumulated number of socioeconomic disadvantages and adolescent's MetS risk score. After adjusting for sex, age, and Tanner stage those adolescents who cumulated three to six disadvantages had a higher MetS score ( $\beta$.68 [99\% CI .07, 1.29]) compared to those with no disadvantages. Although similar results were found in fully adjusted models, additionally adjusting for lifestyle indicators resulted in a statistically significant association ( $\beta .72$ [99\% CI .04, 1.40]) between missing data on socioeconomic disadvantages and having a higher MetS risk score, compared to those with no disadvantages.

\section{Role of lifestyle on MetS risk score}

Additional analyses (results not shown) investigating the associations between lifestyle and MetS showed a positive association between DQI and MetS score and no association between alcohol/smoking and MetS. Only PA was negatively related to MetS score.

\section{Sensitivity analyses}

Our sensitivity analysis yielded similar results when having a categorical outcome instead of a continuous outcome; or when using different definitions of the MetS risk (data only shown when using the World Health Organization cutoffs) (Supplemental Tables S3-S4). Moreover, the associations between socioeconomic disadvantages and MetS risk score were stronger in male adolescents compared to female adolescents 
Table 1

Description of the study population stratified by adolescent's metabolic syndrome (MetS) risk (at risk/not at risk) using World Health Organization (WHO) definition $^{\mathrm{a}}$

\begin{tabular}{|c|c|c|c|c|}
\hline \multirow[t]{2}{*}{ Categorical variables } & \multirow{2}{*}{$\begin{array}{l}\mathrm{N}=1,037 \\
\overline{\mathrm{N}}\end{array}$} & \multicolumn{2}{|c|}{$\begin{array}{l}\text { Metabolic } \\
\text { syndrome } \\
\text { (WHO definition, \%) }\end{array}$} & \multirow[t]{2}{*}{$p$-value } \\
\hline & & $\begin{array}{l}\text { At risk } \\
(\mathrm{n}=328)\end{array}$ & $\begin{array}{l}\text { Not at } \\
\text { risk } \\
(\mathrm{n}=709)\end{array}$ & \\
\hline Age groups & & & & .003 \\
\hline 12.5 to $\leq 15.0$ & 621 & 31.6 & 68.4 & \\
\hline 15.0 to $\leq 17.5$ & 416 & 31.7 & 68.3 & \\
\hline Sex & & & & .010 \\
\hline Male & 489 & 35.6 & 64.4 & \\
\hline Female & 548 & 28.1 & 71.9 & \\
\hline Country & & & & $<.001$ \\
\hline Greece & 98 & 30.6 & 69.4 & \\
\hline Germany & 110 & 48.2 & 51.8 & \\
\hline Belgium & 108 & 16.7 & 83.3 & \\
\hline Crete & 99 & 42.4 & 57.6 & \\
\hline France & 86 & 23.3 & 76.7 & \\
\hline Hungary & 132 & 36.4 & 63.6 & \\
\hline Italy & 95 & 35.8 & 64.2 & \\
\hline Sweden & 96 & 22.9 & 77.1 & \\
\hline Austria & 107 & 29.9 & 70.1 & \\
\hline Spain & 106 & 27.4 & 72.6 & \\
\hline Tanner stage & & & & .115 \\
\hline 4 and 5 & 675 & 31.1 & 68.9 & \\
\hline 1,2 , and 3 & 258 & 29.5 & 70.5 & \\
\hline Missing & 104 & 40.4 & 59.6 & \\
\hline Physical activity & & & & .488 \\
\hline$<1$ hours a day & 179 & 35.2 & 64.8 & \\
\hline $\begin{array}{l}>1 \text { hours a day } \\
\quad \text { (less than } 6 \text { months) }\end{array}$ & 134 & 34.3 & 65.7 & \\
\hline $\begin{array}{l}>1 \text { hours a day } \\
\text { (more than } 6 \text { months) }\end{array}$ & 634 & 30.6 & 69.4 & \\
\hline Missing & 90 & 27.8 & 72.2 & \\
\hline Dietary Quality Index & & & & .149 \\
\hline Low & 399 & 28.3 & 71.7 & \\
\hline High & 398 & 32.7 & 67.3 & \\
\hline Missing & 240 & 35.4 & 64.6 & \\
\hline Smoking & & & & .826 \\
\hline Yes & 195 & 33.8 & 66.2 & \\
\hline Ever smoke & 217 & 32.7 & 67.3 & \\
\hline Never smoke & 605 & 30.6 & 69.4 & \\
\hline Missing & 20 & 30.0 & 70.0 & \\
\hline Alcohol intake & & & & .008 \\
\hline Yes & 181 & 33.7 & 66.3 & \\
\hline No & 599 & 28.0 & 72.0 & \\
\hline Missing & 257 & 38.5 & 61.5 & \\
\hline Mother's education & & & & $<.001$ \\
\hline Low & 347 & 40.3 & 59.7 & \\
\hline Medium or high & 635 & 27.1 & 72.9 & \\
\hline Missing & 55 & 29.1 & 70.9 & \\
\hline Father's education & & & & .006 \\
\hline Low & 359 & 37.0 & 63.0 & \\
\hline Medium or high & 596 & 27.7 & 72.3 & \\
\hline Missing & 82 & 36.6 & 63.4 & \\
\hline Family Affluence Scale & & & & .080 \\
\hline Low & 316 & 34.4 & 64.6 & \\
\hline Medium or high & 721 & 30.0 & 70.0 & \\
\hline Migrant status & & & & .966 \\
\hline Yes & 180 & 31.7 & 68.3 & \\
\hline No & 833 & 31.7 & 68.3 & \\
\hline Missing & 24 & 29.2 & 70.8 & \\
\hline Family structure $^{b}$ & & & & .350 \\
\hline Nontraditional & 266 & 35.0 & 65.0 & \\
\hline Traditional & 737 & 30.7 & 69.3 & \\
\hline Missing & 34 & 26.5 & 73.5 & \\
\hline & & (cont & nued on $n$ & column) \\
\hline
\end{tabular}

Table 1

Continued

\begin{tabular}{|c|c|c|c|c|}
\hline \multirow[t]{2}{*}{ Categorical variables } & \multirow{2}{*}{$\begin{array}{l}\mathrm{N}=1,037 \\
\overline{\mathrm{N}}\end{array}$} & \multicolumn{2}{|c|}{$\begin{array}{l}\text { Metabolic } \\
\text { syndrome } \\
\text { (WHO definition, \%) }\end{array}$} & \multirow[t]{2}{*}{$p$-value } \\
\hline & & $\begin{array}{l}\text { At risk } \\
(\mathrm{n}=328)\end{array}$ & $\begin{array}{l}\text { Not at } \\
\text { risk } \\
(\mathrm{n}=709)\end{array}$ & \\
\hline Parent's employment status & & & & .568 \\
\hline Unemployed & 33 & 33.3 & 66.7 & \\
\hline Non-unemployed & 882 & 31.2 & 68.8 & \\
\hline Missing & 122 & 34.4 & 65.6 & \\
\hline \multicolumn{5}{|l|}{ Number of disadvantages ${ }^{c}$} \\
\hline 3-6 disadvantages & 216 & 39.8 & 60.2 & .008 \\
\hline 2 disadvantages & 183 & 35.5 & 64.5 & \\
\hline 1 disadvantage & 238 & 24.4 & 70.6 & \\
\hline 0 disadvantages & 259 & 25.1 & 74.9 & \\
\hline Missing & 141 & 29.8 & 70.2 & \\
\hline
\end{tabular}

In bold statistically significant differences are shown. Number of participants and percentages (\%) are shown.

Chi-square tests were used to examine differences in the study population by adolescent's MetS risk (at risk/not at risk).

a Risk of Mets defined as having at least of one of the components of metabolic syndrome (obesity, dyslipidemia, hypertension, and glucose intolerance) using the WHO definition.

b Family structure: If the adolescent did not live with both biological parents, the family was defined as a "nontraditional family."

c A total score was calculated by adding up the scores ( 1 vs. 0 ) of the six indicators (low education of the mother, low education of the father, low family affluence (FAS), nontraditional family, migrant background, unemployed). Total score ranges from 0 (the child has none of the six disadvantages indicators) to six (the child has all six disadvantages indicators).

(results shown in Supplemental Table S5). Finally, results did not change when using family affluence as a continuous index instead of a categorical variable.

\section{Discussion}

The mother's education was the most important determinant in adolescent's cardiometabolic health, mainly due to higher levels of glucose intolerance (HOMA-IR) and dyslipidemia (TG and HDL) in case of low education. Those adolescents who did not report socioeconomic information and who accumulated three or more socioeconomic disadvantages had a higher MetS risk than adolescents who did not have any disadvantages. The associations stayed the same before and after adjustment for lifestyle.

\section{Lifestyle-dependent versus lifestyle-independent pathway}

We hypothesized there are two paths through which socioeconomic disadvantages can be associated with MetS, one direct and one indirect. The first pathway is that socioeconomic disadvantages may lead to an unhealthy, MetS increasing lifestyle [30] among others induced by stress (e.g. stress stimulated eating in the absence of hunger, that could facilitate weight gain) or (financial) priority setting. The second identifies associations independent of lifestyle: socioeconomic disadvantages can create a state of continuous stress that increases the activity of the hypothalamic-pituitaryadrenal axis leading to hypercortisolism and hence a higher MetS risk [15]. Since our associations were unchanged 
Table 2

Associations between socioeconomic disadvantages and adolescent's MetS risk score. Results from the linear mixed-effects models: $\beta$ estimates ( $\beta$ ) and 99\% CI results are shown $^{\text {a }}$

\begin{tabular}{|c|c|c|c|c|}
\hline & \multicolumn{2}{|c|}{ MetS risk score (basic adjustment) } & \multicolumn{2}{|c|}{ MetS risk score (full adjustment) ${ }^{c}$} \\
\hline & $\beta$ & $99 \% \mathrm{CI}$ & $\beta$ & $99 \% \mathrm{CI}$ \\
\hline \multicolumn{5}{|c|}{$\begin{array}{l}\text { Mother's education (reference group } \\
\text { medium or high) }\end{array}$} \\
\hline Low & .54 & .09 to $.98 * * *$ & .55 & .10 to $1.00 * * *$ \\
\hline Missing & .18 & -.70 to 1.07 & .18 & -.70 to 1.07 \\
\hline Medium or high & .00 & & .00 & \\
\hline \multicolumn{5}{|c|}{$\begin{array}{l}\text { Father's education (reference group } \\
\text { medium or high) }\end{array}$} \\
\hline Low & .34 & -.09 to .78 & .35 & -.09 to .79 \\
\hline Missing & .74 & .01 to $1.48^{* * *}$ & .98 & .19 to $1.77^{* * *}$ \\
\hline Medium or high & .00 & & .00 & \\
\hline \multicolumn{5}{|c|}{$\begin{array}{l}\text { Family Affluence Scale (reference group } \\
\text { medium or high) }\end{array}$} \\
\hline Low & .17 & -.29 to .63 & .21 & -.26 to .67 \\
\hline Medium or high & .00 & & .00 & \\
\hline \multicolumn{5}{|c|}{$\begin{array}{l}\text { Migrant status }{ }^{\mathrm{d}} \text { (reference group no } \\
\text { migrant) }\end{array}$} \\
\hline Yes & .18 & -.36 to .72 & .21 & -.33 to .76 \\
\hline Missing & -.69 & -2.09 to .72 & -.21 & -1.90 to 1.47 \\
\hline No & .00 & & .00 & \\
\hline \multicolumn{5}{|c|}{$\begin{array}{l}\text { Family structure }^{\mathrm{d}, \mathrm{e}} \text { (reference group } \\
\text { traditional families) }\end{array}$} \\
\hline Nontraditional & .08 & -.36 to .53 & .08 & -.37 to .53 \\
\hline Missing & -.23 & -1.41 to .94 & .25 & -1.10 to 1.60 \\
\hline Traditional & .00 & & .00 & \\
\hline \multicolumn{5}{|c|}{$\begin{array}{l}\text { Parent's employment status }{ }^{\mathrm{d}} \text { (reference } \\
\text { group non-unemployed) }\end{array}$} \\
\hline Unemployed & .78 & -.31 to 1.87 & .86 & -.24 to 1.96 \\
\hline Missing & .24 & -.39 to .86 & .29 & -.35 to .92 \\
\hline Non-unemployed & .00 & & .00 & \\
\hline
\end{tabular}

Results with $* * *$ and in bold indicate $p<.01$.

$\mathrm{CI}=$ confidence interval; MetS $=$ metabolic syndrome.

${ }^{a}$ MetS risk score was calculated as the sum of sex- and age-specific z-scores of BMI, HOMA-IR index, mean of z-scores of diastolic and systolic blood pressure (SBP, DBP), and mean of z-score of HDL cholesterol multiplied by -1 and z-score of triglycerides (TG).

b Basic-models were adjusted for baseline age, sex, and Tanner stage.

c Full models were additionally adjusted Diet Quality Index, physical activity, smoking status, and alcohol consumption.

d Models additionally adjusted for classical SES indicators (maternal education and family affluence measured by FAS).

e Family structure: If the adolescent did not live with both biological parents, the family was defined as a "nontraditional family."

following adjustment for lifestyle, the second lifestyleindependent pathway seems more applicable in our sample. Nevertheless, some of the lifestyle factors were associated with socioeconomic disadvantage and MetS.

\section{Which socioeconomic disadvantages: maternal education and the accumulation}

Only parents' education and particularly mother's education were linked to MetS risk. Importantly, we detected a doseresponse relation as children who accumulated three to six socioeconomic disadvantages were at a higher risk of MetS than children with no disadvantages. A dose-response association related to stress and MetS has been already documented in some previous studies [31], consistent with our result.

The inverse relationship between SES and MetS in developed and developing countries has been shown in previous investigations mostly in adults [6,32]. Studies of parental SES and adolescent's MetS risk have produced contradictory results. Loucks et al. suggested that parental affluence and education were not related to the MetS in adolescents [7]. More in line with our findings, another prospective investigation concluded that family SES in childhood and adolescence was associated with
MetS, impaired fasting glucose or type 2 diabetes in adulthood [8]. It is likely that knowledge, self-efficacy, or motivation towards a healthy lifestyle is more important than financial aspects since only maternal education and not parental unemployment or family affluence was a significant MetS predictor in our study.

Concerning social disadvantages, children and adolescents may not experience this stress directly but indirectly transmitted by their parents through a lack of parenting or quality time that parents could devote to them [33].

Migrant background was not a predictor of adolescent's MetS. Migrant children and adolescents have generally displayed a more sedentary way of life or adverse dietary patterns and higher levels of obesity as compared with native children, though this effect seems to disappear after adjusting for SES [34]. Moreover, lifestyle factors may act in various directions depending on the migrant origin (e.g. diet or PA may be better or worse depending on migrant origin); thus, it is difficult to have a clear hypothesis on the direction of the mediating effect of lifestyle [35].

A nontraditional family structure was also not a significant predictor of adolescent's MetS. Family structure has been suggested to be an important predictor of mental and physical health in children but less in adolescents [36,37]. When children become adolescents, they spend less time at home and peers become more important. 
Table 3

Associations between socioeconomic disadvantages and each component of the adolescent's MetS risk. Fully adjusted models ${ }^{\mathrm{b}}$. Results from the linear mixed-effects models: $\beta$ estimates, 99\% confidence intervals (99\% CI) are shown ${ }^{\mathrm{a}}$

\begin{tabular}{|c|c|c|c|c|c|c|c|c|c|c|c|c|}
\hline & \multicolumn{2}{|c|}{ HOMA-IR z-score } & \multicolumn{2}{|c|}{ BMI z-score } & \multicolumn{2}{|c|}{ SBP z-score } & \multicolumn{2}{|c|}{ DBP z-score } & \multicolumn{2}{|c|}{ HDL z-score } & \multicolumn{2}{|c|}{ TG z-score } \\
\hline & $\beta$ & $99 \% \mathrm{CI}$ & B & $99 \% \mathrm{CI}$ & $\beta$ & $99 \% \mathrm{CI}$ & $\beta$ & $99 \% \mathrm{CI}$ & $\beta$ & $99 \% \mathrm{Cl}$ & $\beta$ & $99 \% \mathrm{CI}$ \\
\hline \multicolumn{13}{|c|}{$\begin{array}{l}\text { Mother's education (reference group } \\
\text { medium or high) }\end{array}$} \\
\hline Low & .19 & .01 to $.38^{* * *}$ & .16 & -.04 to .35 & .07 & -.09 to .33 & -.01 & -.18 to .16 & -.17 & -.37 to $.00^{* * *}$ & .18 & .01 to $.36 * * *$ \\
\hline Missisng & .17 & -.19 to .62 & 17 & -.36 to .49 & 19 & -.37 to .55 & -.07 & -.45 to .31 & -.21 & -.61 to .20 & .19 & -.21 to .58 \\
\hline Medium or high & .00 & & .00 & & .00 & & .00 & & .00 & & .00 & \\
\hline \multicolumn{13}{|c|}{$\begin{array}{l}\text { Father's education (reference group } \\
\text { medium or high) }\end{array}$} \\
\hline Low & .16 & -.02 to .33 & .06 & -.12 to .25 & .10 & -.10 to .30 & -.03 & -.20 to .13 & -.06 & -.24 to .11 & .12 & -.05 to .30 \\
\hline Missing & .26 & -.07 to .57 & .34 & .01 to $.68^{* * *}$ & .24 & -.12 to .61 & .03 & -.26 to .34 & -.29 & -.62 to .03 & .19 & -.13 to .51 \\
\hline Medium or high & .00 & & .00 & & .00 & & .00 & & .00 & & .00 & \\
\hline \multicolumn{13}{|c|}{$\begin{array}{l}\text { Family Affluence Scale (reference group } \\
\text { medium or high) }\end{array}$} \\
\hline Low & .05 & -.14 to .24 & .03 & -.17 to .24 & .16 & -.05 to .38 & -.01 & -.19 to .17 & -.06 & -.25 to .13 & .10 & -.08 to .29 \\
\hline Medium or high & .00 & & .00 & & .00 & & .00 & & .00 & & .00 & \\
\hline \multicolumn{13}{|c|}{$\begin{array}{l}\text { Migrant status }{ }^{\mathrm{C}} \text { (reference group no } \\
\text { migrant) }\end{array}$} \\
\hline Yes & .30 & -.13 to .74 & .09 & -.15 to .32 & .01 & -.21 to .23 & .01 & -.20 to .21 & -.21 & -.42 to .01 & .06 & -.14 to .25 \\
\hline Missing & -.32 & -1.68 to 1.03 & .03 & -.70 to .76 & -.05 & -.74 to .63 & -.26 & -.90 to .37 & -.08 & -.77 to .61 & -.09 & -.80 to .62 \\
\hline No & .00 & & .00 & & .00 & & .00 & & .00 & & .00 & \\
\hline \multicolumn{13}{|c|}{$\begin{array}{l}\text { Family structure }^{\mathrm{cd} d} \text { (reference group } \\
\text { traditional family) }\end{array}$} \\
\hline Nontraditional & .00 & -.19 to .19 & .10 & -.10 to .29 & -.14 & -.35 to .07 & -.02 & -.19 to .15 & -.03 & -.21 to .16 & -.03 & -.22 to .15 \\
\hline Missing & .40 & -.15 to .95 & -.20 & -.79 to .38 & -.36 & -.99 to .26 & -.02 & -.54 to .49 & -.22 & -.78 to .33 & .05 & -.50 to .60 \\
\hline Traditional & .00 & & .00 & & .00 & & .00 & & .00 & & .00 & \\
\hline \multicolumn{13}{|c|}{$\begin{array}{l}\text { Parent's employment status }{ }^{\mathrm{C}} \text { (reference } \\
\text { group non-unemployed) }\end{array}$} \\
\hline Unemployed & .27 & -.18 to .72 & .33 & -.14 to .81 & .15 & -.36 to .66 & .20 & -.22 to .62 & -.17 & -.62 to .29 & -.07 & -.51 to .38 \\
\hline Missing & .03 & -.23 to .30 & .08 & -.20 to .37 & -.18 & -.48 to .12 & -.03 & -.28 to .22 & -.15 & -.40 to .13 & .18 & -.08 to .44 \\
\hline Non-unemployed & .00 & & .00 & & .00 & & .00 & & .00 & & .00 & \\
\hline
\end{tabular}

Results with $* * *$ and in bold indicate $\mathrm{p}<.01$. Statistically significant results considering $99 \% \mathrm{CI}$ are shown in bold font.

$\mathrm{BMI}=$ body mass index; DBP = diastolic blood pressure; HDL = high-density lipoprotein; HOMA-IR = Homeostasis Model Assessment; SBP = systolic blood pressure; TG = triglyceride

${ }^{a}$ All models include random effects (school, country) to account for the study design.

b Models were adjusted for baseline age, sex, Tanner stage, Diet Quality Index, physical activity, smoking status, and alcohol consumption.

" Models additionally adjusted for classical SES indicators (maternal education and family affluence measured by FAS).

d Family structure: If the child did not live with both his/her parents, the family was defined as a "nontraditional family." 
Table 4

Association between the accumulation of socioeconomic disadvantages and MetS risk score in European adolescents ${ }^{\mathrm{a}}$. Results from the linear mixed-effects models: $\beta$ estimates, $99 \%$ confidence intervals (99\% CI) are shown ${ }^{\mathrm{b}}$

\begin{tabular}{|c|c|c|c|c|}
\hline & \multicolumn{2}{|c|}{$\begin{array}{l}\text { MetS risk score (basic } \\
\text { adjustment) }^{\mathrm{b}}\end{array}$} & \multicolumn{2}{|c|}{$\begin{array}{l}\text { MetS risk score } \\
\text { (full adjustment) }^{c}\end{array}$} \\
\hline & $\beta$ & $99 \% \mathrm{CI}$ & $\beta$ & $99 \% \mathrm{CI}$ \\
\hline \multicolumn{5}{|l|}{$\begin{array}{l}\text { Number of } \\
\text { socioeconomic } \\
\text { disadvantages }\end{array}$} \\
\hline 3-6 disadvantages & .68 & .07 to $1.29 * * *$ & .69 & .08 to $1.31^{* * *}$ \\
\hline 2 disadvantages & .41 & -.21 to 1.02 & .45 & -.18 to 1.07 \\
\hline 1 disadvantage & .20 & -.36 to .76 & .19 & -.38 to .75 \\
\hline Missing & .58 & -.08 to 1.24 & .72 & .04 to $1.40^{* * *}$ \\
\hline $\begin{array}{c}0 \text { disadvantages } \\
\text { (reference) }\end{array}$ & .00 & & .00 & \\
\hline
\end{tabular}

Results with $* * *$ and in bold indicate $p<.01$.

MetS $=$ metabolic syndrome.

All models include random effects (school, country) to account for the study design.

a MetS risk score was calculated as the sum of sex- and age-specific z-scores of BMI, HOMA-IR index, mean of z-scores of diastolic and systolic blood pressure (SBP, DBP), and mean of z-score of HDL cholesterol multiplied by -1 and z-score of triglycerides (TG).

b Basic models were adjusted for baseline age, sex, and Tanner stage.

c Fully adjusted models were additionally adjusted for Diet Quality Index, physical activity, smoking status, and alcohol consumption.

${ }^{d}$ A total score was calculated by adding up the scores ( 1 vs. 0 ) of the six indicators (low education of the mother, low education of the father, low family affluence (FAS), nontraditional family, migrant background, unemployed). Total score ranges from 0 (the child has none of the six disadvantages indicators) to six (the child has all six disadvantages indicators).

\section{Different impact by sex}

This investigation also studied whether the association between socioeconomic disadvantages and MetS in adolescents differed by sex. Some studies have shown a strong association between SES and MetS in females and less strong in males [6]. Possible mechanisms include the more severe association between SES and obesity (strongly related with MetS) in females than in males and the greater exposure to adverse psychosocial conditions such as depression and anxiety among women from low SES that in turn may lead to higher risk of MetS [38]. Our findings are in disagreement with previous literature possibly due to the higher percentage of MetS risk observed in males than in females.

\section{Strengths and limitations}

To our knowledge this is the first study to investigate the relationship between socioeconomic disadvantages and MetS risk in a large sample of European adolescents independent of lifestyle indicators. The strengths of this study include the large sample of adolescents of different ages with blood samples using a standardized methodology across the participating cities and the use of sex and age-dependent continuous and categorical MetS risk indicators based on different definitions.

This study has also some limitations. Owing to the crosssectional design, causal conclusions cannot be inferred [39]. Missing data in some subgroups and relying on self-reports of lifestyle factors are further limitations. Dietary variables are prone to error [40] and the use of only two $24 \mathrm{~h}$ recalls might not capture some alcohol drinking patterns such as binge drinking. Such reporting/measurement error might also be the reason for our observation of a higher DQI in those with a higher MetS risk; overweight people tend to underreport or report healthier diets [41], although it is possible that they are following a weight loss diet. Moreover, this study is not representative of each European country included although schools and classes were randomly selected allowing to represent the situation of European adolescents living in urban areas. Finally, the lack of significant results may be due to the heterogeneity of some groups in this study. For example, migrant origin and reasons for migration may be different from one person to another and nontraditional families include various situations (one-parent families, living with other relatives/friends or in institutions), and consequently some groups could be more vulnerable than others [42].

Maternal education was the most important determinant of adolescent's MetS risk independent of sex, age, Tanner stage, and lifestyle. Social vulnerabilities (migrant background, unemployment status, and belonging to a nontraditional family) were not associated with a higher MetS risk in European adolescents; however, we found a dose-response relationship between the number of social disadvantages and adolescents' MetS risk. Psychosocial stressors derived from psychosocial disadvantages can be one of the reasons to explain the higher MetS found in adolescents with low-educated mothers or with a higher accumulated number of vulnerabilities. Policy makers should focus on low-educated families and more disadvantaged groups to improve their knowledge of nutrition, exercise, and strategies to cope with stress to tackle health disparities.

\section{Acknowledgments}

The authors would like to express our great appreciation to all the children and parents who participated in this study. They also thank Tracy Lignini for the English editing service. The present paper was presented as a poster in the 13th European Nutrition Conference in Dublin (Ireland).

\section{Funding Sources}

The HELENA Study was conducted with the financial support of the European Community sixth RTD Framework Programme (Contract FOOD-CT-2005-007034).

\section{Supplementary Data}

Supplementary data related to this article can be found at https://doi.org/10.1016/j.jadohealth.2020.05.027.

\section{References}

[1] Ford ES. Risks for all-cause mortality, cardiovascular disease, and diabetes associated with the metabolic syndrome: A summary of the evidence. Diabetes Care 2005;28:1769-78.

[2] Nolan PB, Carrick-Ranson G, Stinear JW, et al. Prevalence of metabolic syndrome and metabolic syndrome components in young adults: A pooled analysis. Prev Med Rep 2017;7:211-5.

[3] Saklayen MG. The Global Epidemic of the metabolic syndrome. Curr Hypertens Rep 2018;20:12.

[4] Miller JM, Kaylor MB, Johannsson M, et al. Prevalence of metabolic syndrome and individual criterion in US adolescents: 2001-2010 national health and nutrition examination survey. Metab Syndr Relat Disord 2014; 12:527-32.

[5] Kaur J. A comprehensive review on metabolic syndrome. Cardiol Res Pract 2014;2014:943162.

[6] Loucks EB, Rehkopf DH, Thurston RC, et al. Socioeconomic disparities in metabolic syndrome differ by gender: Evidence from NHANES III. Ann Epidemiol 2007;17:19-26. 
[7] Loucks EB, Magnusson KT, Cook S, et al. Socioeconomic position and the metabolic syndrome in early, middle, and late life: Evidence from NHANES 1999-2002. Ann Epidemiol 2007;17:782-90.

8] Puolakka E, Pahkala K, Laitinen TT, et al. Childhood socioeconomic status in predicting metabolic syndrome and glucose abnormalities in adulthood: The cardiovascular risk in young Finns study. Diabetes Care 2016;39:2311-7.

[9] Iguacel I, Michels N, Ahrens W, et al. Prospective associations between socioeconomically disadvantaged groups and metabolic syndrome risk in European children. Results from the IDEFICS study. Int J Cardiol 2018;272: 333-40.

[10] Park HS, Oh SW, Cho SI, et al. The metabolic syndrome and associated lifestyle factors among South Korean adults. Int J Epidemiol 2004;33: $328-36$.

[11] Candib LM. Obesity and diabetes in vulnerable populations: Reflection on proximal and distal causes. Ann Fam Med 2007;5:547-56.

[12] Suglia SF, Koenen KC, Boynton-Jarrett R, et al. Childhood and adolescent adversity and cardiometabolic outcomes: A scientific statement from the American Heart association. Circulation 2018;137:e15-28.

[13] Lovasi GS, Hutson MA, Guerra M, et al. Built environments and obesity in disadvantaged populations. Epidemiol Rev 2009;31:7-20.

[14] Poulton R, Caspi A. Commentary: How does socioeconomic disadvantage during childhood damage health in adulthood? Testing psychosocial pathways. Int J Epidemiol 2005;34:344-5.

[15] Anagnostis P, Athyros VG, Tziomalos K, et al. Clinical review: The pathogenetic role of cortisol in the metabolic syndrome: A hypothesis. J Clin Endocrinol Metab 2009;94:2692-701.

[16] Olsson CA, Romaniuk H, Salinger J, et al. Drinking patterns of adolescents who develop alcohol use disorders: Results from the Victorian adolescent health cohort study. BMJ Open 2016;6:e010455.

[17] Béghin L, Huybrechts I, Vicente-Rodríguez G, et al. Main characteristics and participation rate of European adolescents included in the HELENA study. Arch Public Health 2012;70:1-11.

[18] Bammann K, Gwozdz W, Lanfer A, et al. Socioeconomic factors and child hood overweight in Europe: Results from the multi-centre IDEFICS study. Pediatr Obes 2013;8:1-12.

[19] Boyce W, Torsheim T, Currie C, et al. The family affluence scale as a measure of national Wealth: Validation of an adolescent self-report measure. Social Indicators Res 2006;78:473-87.

[20] Iguacel I, Fernandez-Alvira JM, Bammann K, et al. Associations between social vulnerabilities and dietary patterns in European children: The identification and prevention of dietary- and lifestyle-induced health EFfects in children and infantS (IDEFICS) study. Br J Nutr 2016;116:1288-97.

[21] Iguacel I, Michels N, Fernandez-Alvira JM, et al. Associations between socia vulnerabilities and psychosocial problems in European children. Results from the IDEFICS study. Eur Child Adolesc Psychiatry 2017;26:1105-17.

[22] Nagy E, Vicente-Rodriguez G, Manios Y, et al. Harmonization process and reliability assessment of anthropometric measurements in a multicenter study in adolescents. Int J Obes (Lond) 2008;32:S58-65.

[23] Gonzalez-Gross M, Breidenassel C, Gomez-Martinez S, et al. Sampling and processing of fresh blood samples within a European multicenter nutritional study: Evaluation of biomarker stability during transport and storage. Int J Obes (Lond) 2008;32:S66-75.

[24] Tanner JM, Whitehouse RH. Clinical longitudinal standards for height weight, height velocity, weight velocity, and stages of puberty. Arch Dis Child 1976;51:170-9.

[25] Ahrens W, Moreno LA, Marild S, et al. Metabolic syndrome in young children: Definitions and results of the IDEFICS study. Int J Obes (Lond) 2014; 38:S4-14.

[26] Vyncke K, Cruz Fernandez E, Fajo-Pascual M, et al. Validation of the die quality index for adolescents by comparison with biomarkers, nutrient and food intakes: The HELENA study. Br J Nutr 2013;109:2067-78.

[27] Kim S, Egerter S, Cubbin C, et al. Potential implications of missing income data in population-based surveys: An example from a Postpartum survey in California. Public Health Rep 2007;122:753-63.

[28] Vanlancker T, Schaubroeck E, Vyncke K, et al. Comparison of definitions for the metabolic syndrome in adolescents. The HELENA study. Eur J Pediatr 2017:176:241-52.

[29] IBM Corp. Released. IBM SPSS Statistics for Windows, version 24.0. Armonk, NY: IBM Corp; 2016.

[30] Michels N, Sioen I, Boone L, et al. Longitudinal association between child stress and lifestyle. Health Psychol 2015;34:40-50.

[31] Elsenburg LK, van Wijk KJE, Liefbroer AC, et al. Accumulation of adverse childhood events and overweight in children: A systematic review and meta-analysis. Obesity (Silver Spring) 2017;25:820-32.

[32] Park HS, Oh SW, Cho SI, et al. The metabolic syndrome and associated life style factors among South Korean adults. Int J Epidemiol 2004:33:328-36.

[33] Tamashiro KL. Metabolic syndrome: Links to social stress and socioeconomic status. Ann N Y Acad Sci 2011;1231:46-55.

[34] Liu J, Probst JC, Harun N, et al. Acculturation, physical activity, and obesity among Hispanic adolescents. Ethn Health 2009;14:509-25.

[35] Darmon N, Khlat M. An overview of the health status of migrants in France, in relation to their dietary practices. Public Health Nutr 2001;4:163-72.

[36] Xu YQ, Ji CY. Prevalence of the metabolic syndrome in secondary school adolescents in Beijing, China. Acta Paediatr 2008;97:348-53.

[37] Iguacel I, Fernandez-Alvira JM, Ahrens W, et al. Prospective associations between social vulnerabilities and children's weight status. Results from the IDEFICS study. Int J Obes (Lond) 2018;42:1691-703.

[38] Wu HF, Tam T, Jin L, et al. Age, gender, and socioeconomic gradients in metabolic syndrome: Biomarker evidence from a large sample in Taiwan, 2005-2013. Ann Epidemiol 2017;27:315-322.e312.

[39] Grimes DA, Schulz KF. Descriptive studies: What they can and cannot do. Lancet 2002;359:145-9.

[40] Freedman LS, Schatzkin A, Midthune D, et al. Dealing with dietary measurement error in nutritional cohort studies. J Natl Cancer Inst 2011;103 1086-92.

[41] Little P, Barnett J, Margetts B, et al. The validity of dietary assessment in general practice. J Epidemiol Community Health 1999;53:165-72.

[42] Stronks K, Kulu-Glasgow I, Agyemang C. The utility of 'country of birth' for the classification of ethnic groups in health research: The Dutch experience. Ethn Health 2009;14:255-69. 